\title{
Zur vorliegenden Buess-Festschrift
}

Zuerst wurde eine Namenliste aufgesetzt. Wer sollte um einen Beitrag gebeten werden? Vorab die Basler: die langjährige unentbehrliche Mitarbeiterin am Balser Medizinhistorischen Institut, Fräulein Dr. Marie-Louise Portmann. Sie steuerte auch das Verzeichnis der Publikationen bei. Dann die neu hinzugekommenen dortigen Mitarbeiter: Ehrendozent René Bernoulli und Privatdozent Ulrich Tröhler. Der ehemalige Doktorand Werner Stöcklin, der als Arzt und Ethnograph weiterarbeitet. Drei Kollegen: Prof. Friedrich Rintelen, der fesselnde Lehrer, Ophthalmologe und Medizinhistoriker, Dr. Hans Leonhard Küng, Fabrikarzt und Lehrbeauftragter für Arbeitsmedizin, Prof. Heinrich Stamm, gynäkologischer Chefarzt in Baden. Auch der Veterinär Werner Sackmann in Riehen sagte einen Beitrag zu.

Vom Zürcher Institut kamen in Frage: der jetzige Leiter, der sich bei Buess habilitiert hat, Prof. Koelbing, Altmeister Ackerknecht, Professor Walser, Museumskonservator Dr. Urs Boschung.

Dazu gab es einen Kreis alter Freunde im Ausland: Prof. Nikolaus Mani in Bonn, der Bündner Medizinhistoriker, der sich als erster bei Professor Buess habilitiert hatte, Professor Rothschuh in Münster, Professor Schipperges in Heidelberg, in Österreich die um fünf Tage jüngere Frau Professor Lesky, in Italien Belloni und Premuda.

Da die Zeit knapp war, erwartete ich viele Absagen. Statt dessen schneite es Manuskripte. Die Begleitbriefe sprachen die Gesinnung aus: Wenn es um Professor Buess geht, kann man nicht nein sagen. Mit Bangen sah ich, wie das Heft immer dicker wurde. Ich wagte nicht mehr, Professor Robert Blaser in Neuenburg, Professor Schadewaldt in Düsseldorf, Frau Professor Putscher in Köln, Dr. Gerhard Grosch in Königstein im Taunus und Professor Walter Pagel anzufragen, den Gelehrten in London, für den Professor Buess einst den Basler Ehrendoktortitel erwirkt hat. Frau Leskys Beitrag «Der angeklagte Gall», mein eigener über «Die Entdeckung der Eiszeit» werden auf das nächste Heft verschoben. Wer durch Umstände am Schreiben verhindert war, schickt liebe Grüße, so von Bern Frau Professor FischerHomberger und Paul Röthlisberger. Dafür trafen, teils unerwartet, noch andere Beiträge ein: von Frau Meyer-Salzmann, von Frau Hansch-Mock, von Isaac Benguigui, von Hanno Beck, von René Renggli. 
Es galt, sie in eine Reihe zu ordnen. Am Beginn stehen würdigende Worte und die Bibliographie. Da Professor Buess von der Gynäkologie ausgegangen war (sein ältester Sohn ist ebenfalls Gynäkologe geworden und wirkt am Spital Sumiswald), zeichnete sich als erster Schwerpunkt die Gynäkologie ab. Um diesen Kern lagern sich die Beiträge von Stamm, Koelbing, Boschung, Tröhler. (Herr Professor Stamm übernahm ohne Zögern die Herstellungskosten aller seiner Abbildungen.) Es folgen Basiliensia von Marie-Louise Portmann und Friedrich Rintelen. Allgemeine Aspekte der Medizingeschichte besprechen Schipperges, Bernoulli, Ackerknecht. Die nächste Gruppe vereinigt Arbeiten zu besonderen Fragen in zeitlicher Folge vom 16. bis zum 20. Jahrhundert: Belloni, Mani, Frau Meyer-Salzmann, Frau Hansch-Mock, Rothschuh, Premuda, Walser, Sackmann. Instrumentelles behandeln Benguigui, Stöcklin und Renggli. Einen Schlußakkord gibt Hanno Beck.

Heinz Balmer 